\title{
A-dependence of the Beam-Spin Azimuthal Asymmetry in Deeply Virtual Compton Scattering
}

\author{
Hayg Guler (on behalf of the HERMES collaboration)
}

DESY, D-15718 Zeuthen, Germany

\begin{abstract}
The nuclear-mass dependence of the beam-spin asymmetry (BSA) in deeply virtual Compton scattering has been measured at HERMES. The BSA ratios of Nuclei to Hydrogen or Deuterium have been extracted in coherent and incoherent-enriched kinematic regions separately.
\end{abstract}

\section{Introduction}

Lepton scattering experiments constitute an important source of information for the understanding of nucleon and nucleus structure. Until recently, this structure was described by two non-perturbative objects, form factors (FFs) and parton distribution functions (PDFs), which were measured in elastic and deep inelastic scattering (DIS) experiments, respectively. In the last decade, Generalized Parton distributions (GPDs) were recognized as a tool to give a unified description of hard exclusive processes in the Bjorken regime, i.e. for large transfers of squared four-momentum $Q^{2}$, and energy $\mathrm{v}$, of the exchanged virtual photon. The GPD formalism offers a much more complete description of nucleon structure than the well-known PDFs and FFs [2,3]. There exist four leading-twist GPDs for each quark species in the nucleon: $H, E, \widetilde{H}$, and $\widetilde{E}$. GPDs allow to access the 3 -dimensional structure of the nucleon [2]. They depend upon three kinematic quantities: the longitudinal momentum fraction of initial and final quarks, $x+\xi$ and $x-\xi(\xi$ being the longitudinal momentum asymmetry or skewness), and the reduced four-momentum squared transfer $t^{\prime}$ to the target.

\section{Deeply Virtual Compton Scattering}

Hard exclusive lepto-production of a real photon, deeply virtual Compton scattering (DVCS), is known to be one of the experimentally cleanest and presently the most practical way to access GPDs. This process has the same initial and final state as the Bethe-Heitler $(\mathrm{BH})$ process, in which the real photon is radiated from the incoming or scattered lepton. As the two processes are experimentally indistinguishable, their amplitudes add coherently and the cross section contains an interference term $I$ :

$$
\frac{d \sigma}{d x_{B} d Q^{2} d\left|t^{\prime}\right| d \phi} \propto\left|\tau_{D V C S}\right|^{2}+\left|\tau_{B H}\right|^{2}+I ; \quad I=\tau_{B H}^{*} \tau_{D V C S}+\tau_{D V C S}^{*} \tau_{B H}
$$

Here $x_{B}=\frac{Q^{2}}{2 M v}$ represents the Bjorken scaling variable. The azimuthal angle $\phi$ is defined as the angle between the lepton scattering plane, spanned by incoming and scattered leptons, and the photon production plane, defined by virtual and real photons. Although at HERMES energies the BH cross section dominates over that of DVCS, the DVCS amplitude can be studied via the interference term $I$, by measuring various azimuthal cross section asymmetries. At leading twist, the interference term can be expanded in terms of Fourier moments in $\phi[3]$ :

$$
I \propto \pm\left(c_{0}^{I}+\sum_{n=1}^{3} c_{n}^{I} \cos (n \phi)+\lambda \sum_{n=1}^{3} s_{n}^{I} \sin (n \phi)\right)
$$


where the $+(-)$ sign stands for a negatively (positively) charged lepton beam, and $\lambda$ is its longitudinal polarization. The coefficients $c_{1}^{I}$ and $s_{1}^{I}$ are proportional to the real and the imaginary part of the DVCS helicity amplitude $M^{1,1}$, respectively. In the case of an unpolarized proton target, this amplitude is given by a linear combination of the complex Compton Form Factors (CFFs), $\mathcal{H}, \tilde{\mathcal{H}}$ and $\mathcal{E}$, together with the known Dirac and Pauli elastic form factors $F_{1}$ and $F_{2}$ :

$$
M^{1,1}=F_{1} \mathcal{H}+\frac{x_{B}}{2-x_{B}}\left(F_{1}+F_{2}\right) \widetilde{\mathcal{H}}-\frac{t}{4 M^{2}} F_{2} \mathcal{E}
$$

The CFFs are convolutions of the respective twist-2 GPDs with hard scattering kernels.

Nuclear targets are studied to learn about the DVCS process in the more complicated nuclear environment. For a nuclear target there exist two distinct processes:

- the coherent process, in which the scattering occurs on the whole nucleus, which stays intact after the emission of a real photon;

- the incoherent process, where the nucleus breaks up, and the real photon is emitted by a particular proton or neutron.

\section{DVCS at HERMES}

HERMES is a fixed-target experiment using the $27.6 \mathrm{GeV}$ longitudinally polarized electron or positron beam of the HERA collider and an internal gas target that can be filled with polarized $\mathrm{H}, \mathrm{D}$ and unpolarized nuclei ( $\mathrm{N}, \mathrm{He}, \mathrm{Ne}, \mathrm{Xe}, \mathrm{Kr}$ ). The DVCS process is measured by identifying the scattered lepton and the produced real photon in the forward spectrometer [4]. As the recoil proton or nucleus can not be detected there, kinematic requirements are imposed in order to ensure the exclusivity of the reaction. In particular, the missing mass is required to be in the range $-1.5<M_{x}<1.7$ $\mathrm{GeV}$, determined from Monte-Carlo simulations by comparing signal to background distributions taking into account the finite resolution of the spectrometer.

\subsection{A-dependence of the Beam-Spin Asymmetry}

The beam-spin asymmetry (BSA), as a function of the azimuthal angle $\phi$, is calculated as

$$
A_{L U}(\phi)=\frac{1}{\left\langle\left|P_{l}\right|\right\rangle} \frac{\vec{N}(\phi)-\overleftarrow{N}(\phi)}{\vec{N}(\phi)+\overleftarrow{N}(\phi)}
$$

with the luminosity normalized yields $\vec{N}(\overleftarrow{N})$ using a beam with positive (negative) helicity, $P_{l}$ being the beam polarization and $\mathrm{L}(\mathrm{U})$ meaning longitudinally polarized beam (unpolarized target). In leading order $\alpha_{S}$ and at leading twist, the $\sin \phi$ amplitude of the $\mathrm{BSA}, A_{L U}^{\sin \phi}$, is proportional to Im $M^{1,1}$. Azimuthal asymmetries with respect to the beam spin have been measured on $\mathrm{H}, \mathrm{D}, \mathrm{He}$, $\mathrm{N}, \mathrm{Ne}, \mathrm{Kr}$ and $\mathrm{Xe}$. For the three targets Deuterium [5] Neon and Krypton, preliminary BSA results integrated over the experimental acceptance are similar to that for the proton [5]. Events can be separated into coherent and incoherent-enriched samples corresponding to separate intervals in $t^{\prime}$. Here these samples are extracted by target-dependent requirements on $t^{\prime}$ in order to provide the same value of $\left\langle t^{\prime}\right\rangle$ for each target. The resulting values are:

- for the coherent-enriched sample: $\left\langle-t^{\prime}\right\rangle=0.018 \mathrm{GeV}^{2}$

- for the incoherent-enriched sample: $\left\langle-t^{\prime}\right\rangle=0.2 \mathrm{GeV}^{2}$. 


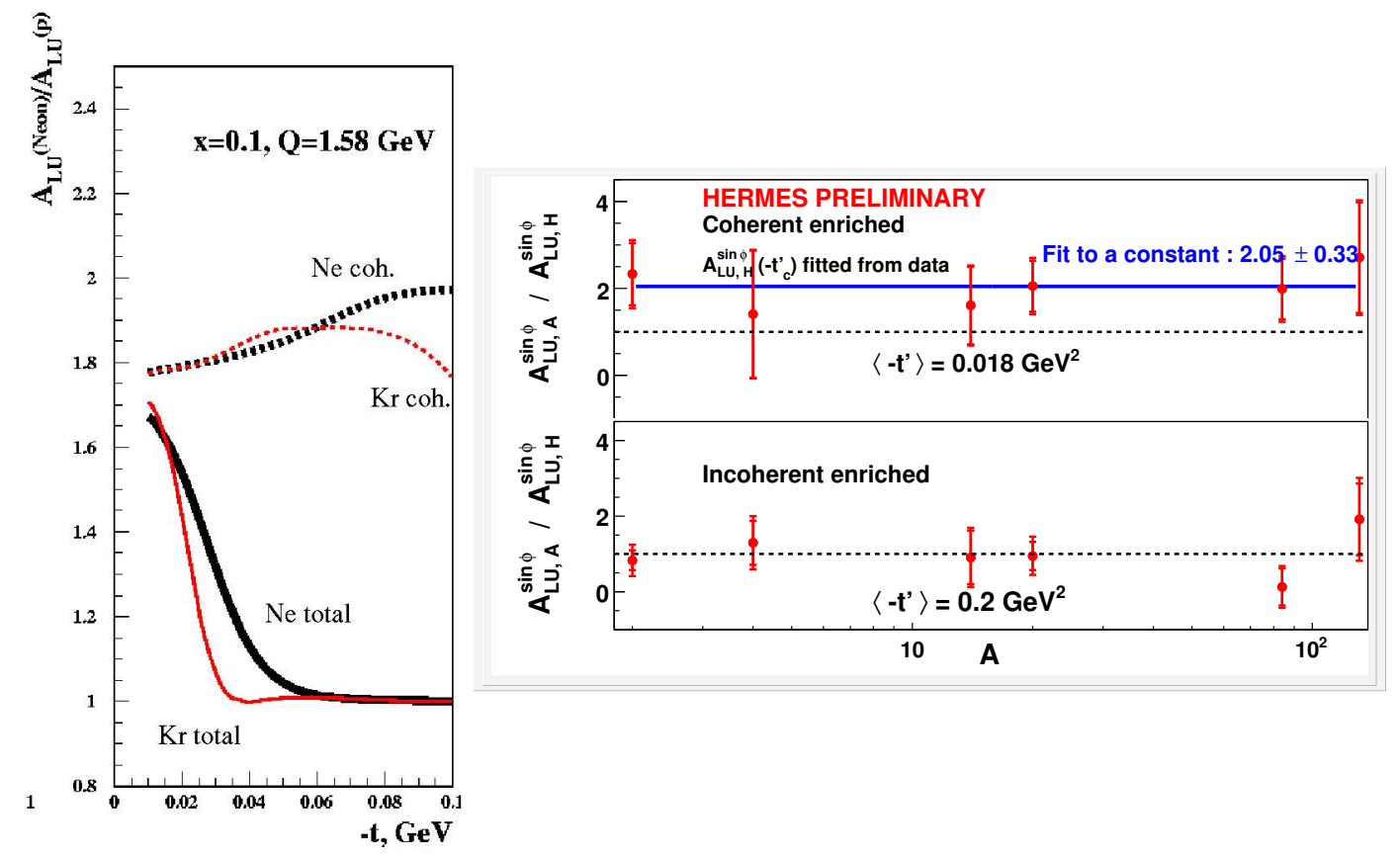

Figure 1: Left panel: model predictions for the ratio of nuclear-to-proton BSA for Neon and Krypton. Right panel: BSA ratio vs. A relative to Hydrogen data fit at $\left\langle-t^{\prime}\right\rangle=0.018 \mathrm{GeV}^{2}$. The dashed line denotes unity and the solid line represents the result of a fit to a constant.

At small $\left\langle t^{\prime}\right\rangle$ the Hydrogen sample has limited statistics, and hence dominates the uncertainties of the BSA ratios. The alternative is to use a fit of the hydrogen BSA anchored by $A_{L U}^{\sin \phi}=0$ at $t^{\prime}=0$, based on the theoretical expectation: $A_{L U}^{\sin \phi}\left(t^{\prime}\right) \propto \sqrt{-t^{\prime}}$ at small $t^{\prime}$. The fit function has the form:

$$
A_{L U}^{\sin \phi}\left(t^{\prime}\right)=\frac{a \cdot \sqrt{-t^{\prime}}}{1+b \cdot{\sqrt{-t^{\prime}}}^{3}},
$$

with the parameters : $a=-1.204 \mathrm{GeV}^{-1}$ and $b=35 \mathrm{GeV}^{-3}$

evaluated at $\left\langle-t^{\prime}\right\rangle=0.018 \mathrm{GeV}^{2}$

The extracted ratios of nuclear-to-hydrogen $A_{L U}^{\sin \phi}$ amplitudes for the coherent and incoherent-enriched samples are shown in the right panel of Fig. 1. The mean ratio in the coherent region deviates from unity by $2 \sigma$ and is consistent with model predictions based on GPD models [8]. As shown in the left panel, they predict for Neon and Krypton a ratio to hydrogen close to 1.8 in the coherent region and consistent with unity in the incoherent one. For the incoherent-enriched sample the mean ratio is also consistent with unity as predicted by the model.

\section{Acknowledgments}

This work has been supported by the German Bundesministerium für Bildung und Forschung (BMBF) (contract nr. 06 ER 125I) and the European Community-Research Infrastructure Activity under the 
FP6 "Structuring the European Research Area" program (HadronPhysics I3, contract nr. RII3-CT2004-506078).

\section{References}

[1] Slides:

http: / / indico. cern. ch/contributionDisplay.py? contribId=87\&sessionId=12\& confId=9499

[2] M. Burkardt, Phys. Rev. D62 (2000) 071503; Erratum-ibid. D 66 (2002) 119903.

[3] A.V. Belitsky and D. Müller, Nucl. Phys. A711 (2002) 118.

[4] HERMES Coll., K. Ackerstaff et al., Nucl. Instr. and Meth. A417 (1998) 230.

[5] F. Ellinghaus et al., for the HERMES Coll., arXiv:hep-ex/0212019, AIP Conf. Proc. 675 (2002) 303, Eds. I. Y. Makdisi et al..

[6] D. Müller et al., Fortschr. Phys. 42 (1994) 101.

[7] X. Ji, Phys. Rev. Lett. 78 (1997) 610, Phys. Rev. D55 (1997) 7114.

[8] V. Guzey and M. Strikman, Phys. Rev. C68 (2003) 015204.

[9] A.V. Radyushkin, Phys. Rev. D56 (1997) 5524.

[10] K. Goeke et al., Prog. Part. Nucl. Phys. 47 (2001) 401. 